\title{
Specialist Aphid Feeding Causes Local Activation of Salicylic and Jasmonic Acid Signaling in Arabidopsis Veins
}

\author{
Nikoleta Rubil, ${ }^{1,2,3}$ Tetiana Kalachova, ${ }^{1, \dagger}$ Thure Pavlo Hauser, ${ }^{3}$ and Lenka Burketová ${ }^{1}$ \\ ${ }^{1}$ Institute of Experimental Botany, The Czech Academy of Sciences, Rozvojová 263, 165 02, Prague 6, Czech Republic \\ ${ }^{2}$ Czech University of Life Sciences, Kamýcká 129, 16500 Prague 6-Suchdol, Czech Republic \\ ${ }^{3}$ Department of Plant and Environmental Sciences, Copenhagen University, Thorvaldsensvej 40, 1871 Frederiksberg C, \\ Denmark
}

Accepted 19 October 2021.

\begin{abstract}
Aphids, the phloem sap feeders, probe into leaf tissues and activate a complex network of plant defense responses. Phytohormonal signaling plays a major role in this network; however, the dynamics of the signal spreading is yet to be clarified. Despite the growing knowledge about transcriptomic changes upon infestation, results often differ due to sampling, varying strongly between the tissues collected at the single feeding site, individual leaves, pooled infested leaves, or whole plant rosettes. This study focuses on activation of salicylic acid (SA) and jasmonic acid (JA) signals in Arabidopsis leaves during infestation by cabbage aphid (Brevicoryne brassicae) in high spatio-temporal resolution. We used genetically encoded fluorescent biosensors, histochemistry, and quantitative reverse transcription-PCR to precisely map activation of distinct branches of phytohormonal signaling. We found a rapid induction of SA and JA signaling markers in cells surrounding stylet puncture, colocalizing with callose deposition. For both $P R I$ and JAZ10, we detected activation at $24 \mathrm{~h}$ postinfestation (hpi), increasing and spreading along the veins until $72 \mathrm{hpi}$ and, to a lesser extent, within the epidermal pavement cells. The $\mathrm{SA}$ signaling wave appeared in parallel with $\mathbf{J A}$-associated signaling and continued to increase in time. Our results first show a local activation of SA- and JA-related responses after stylet penetration of Arabidopsis leaves and bring a detailed insight into the spatiotemporal complexity of plant defense activation during specialist aphid attack.
\end{abstract}

Keywords: Arabidopsis thaliana, Brevicoryne brassicae, callose, infestation, jasmonic acid, salicylic acid, phytohormonal signaling

Plants are frequently attacked by herbivores and pathogens and have evolved complex defense mechanisms to counter this,

${ }^{\dagger}$ Corresponding author: T. Kalachova; kalachova@ueb.cas.cz

Funding: This study was funded by Ministry of Education, Youth and Sports of Czech Republic (Ministerstvo školství, mládeže a tělovýchovy) grants LM2018129 and LTC17013 and European Regional Development Fund grant CZ.02.1.01/0.0/0.0/16_019/0000738.

*The $\boldsymbol{e}$-Xtra logo stands for "electronic extra" and indicates there are supplementary materials published online.

The author(s) declare no conflict of interest. distributed under the CC BY-NC-ND 4.0 International license. including localized as well as remote reactions. The immediate local responses are often accompanied by spreading signals to induce more remote reactions; however, the dynamics of these signaling responses are still not understood in detail. As phloem sap feeding insects, aphids evolved a characteristic elongated mouthpart, called a stylet, by which they probe into plant tissue, secrete saliva, and, finally, reach for the nutrients inside the sieve elements. For successful colonization, specialized molecules in aphid saliva, termed effectors, manipulate plant defense responses during probing and feeding periods. During probing, the stylet follows an intercellular pathway in the mesophyll while excreting gelling saliva. Many cells are punctured intracellularly along the stylet track and are filled with watery saliva (Miles 1959). Stylets are immediately retracted in the moment of cell puncture and continue to move between the cells in the direction of sieve elements, aiming to avoid the activation of plant defense mechanisms (Miles 2007; Tjallingii 2006). During the stylet penetration into sieve elements, aphids start a massive secretion of watery salivary molecules, with the goal to prevent sieve-tube occlusion by interacting with calcium ions (Miles 2007; Tjallingii 2006; Will et al. 2009). Hence, when a stylet finally punctures phloem cells, $\mathrm{Ca}^{2+}$ increases in these and triggers protein and callose synthesis, which occludes sieve tubes to prevent loss of phloem sap (Knoblauch and van Bel 1998; Silva-Sanzana et al. 2020). In response to this plant reaction, the attacker tries to manipulate plant defense responses by injecting $\mathrm{Ca}^{2+}$-binding proteins, with the goal to suppress the blockage of nutrients (Tjallingii 2006; Will et al. 2007, 2009). However, the host plant may be able to recognize aphid-derived molecules or elicitors, resulting in an activation of a complex series of molecular defense responses, such as triggering of intracellular signaling cascades in which phytohormones, e.g., salicylic acid (SA), jasmonic acid (JA), and ethylene (ET), play major roles (Jaouannet et al. 2014; Kuśnierczyk et al. 2008).

Up to now, the spatial dynamics of defense activation in leaf tissues in response to aphid feeding remains rather enigmatic. Despite an increasing amount of data, the sensitivity and accuracy of transcriptome studies is largely limited by various factors: i) sample preparation, as a certain amount of leaf tissues needs to be homogenized, ii) highly heterogeneous infestation due to the distinct number of individuals feeding on the leaf, and iii) different methods of sampling between studies. Several studies reported the induction of SA and JA signaling responses during the aphid infestation (De Vos et al. 2005; Moran and Thompson 2001), while attackers differing in a range of host plants, i.e., specialists or generalists, trigger distinct changes in plant transcriptome (Kuśnierczyk et al. 2007; Mewis et al. 2005; 
Moran et al. 2002). On the other hand, some of the studies reported changes in gene expression when sampling the whole rosettes of Arabidopsis (Kuśnierczyk et al. 2008; Mewis et al. 2005; Onkokesung et al. 2019) or the individual aphid-infested leaves (Kuśnierczyk et al. 2007; Moran and Thompson 2001; Moran et al. 2002). However, feeding aphids are not homogenously dispersed on the leaf but mostly localize along the veins, which might introduce a significant bias into sampling.

Our goal was to investigate the dynamics of Arabidopsis thaliana defense responses during the infestation with a specialist aphid, Brevicoryne brassicae, in higher spatial resolution, with a special focus on the cells in close contact with the stylet. For this, we used a combination of biochemical and molecular biology methods with novel visualization tools to dissect the role of individual cell reactions and cell-to-cell communication in response to herbivorous attacks. To study the spatial progression of the signal induced by feeding aphids, we combined the following approaches: i) quantitative reverse transcription PCR (qRT-PCR) of marker genes for different signaling pathways, sampling 6-mm leaf discs around the aphid feeding site; ii) monitoring activation of marker genes in vivo in the area surrounding the feeding site, using a set of visualization tools, including plant lines expressing promoter-fluorescent reporter constructs for genes associated with SA, JA, and ET signaling pathways (Marhavý et al. 2019); and iii) monitoring the localization of $P R I$ activation using plants expressing the PR1::GUS construct.

Four-week-old $A$. thaliana plants were cultivated in a short-day photoperiod before infestation with $B$. brassicae, and samples were collected at $6,24,48$, and $72 \mathrm{~h}$ postinfestation (hpi). Based on previously published results about transcriptome remodeling upon infestation, several genes marking activation of distinct hormone signaling pathways were chosen for qRT-PCR: SA (PATHOGENESIS-RELATED 1, 2, and 5 [PR1, PR2, PR5], ISOCHORISMATE SYNTHASES 1 and 2 [ICS1, ICS2]), JA (LIPOXYGENASE 2 [LOX2], Allene Oxide Synthase [AOS], Jasmonate ZIM-Domain 8 and 10 [JAZ8, JAZ10]), ET (ACC SYNTHASES 2 and 6 [ACS2, ACS6]), and ET/JA (PLANT DEFENSIN 1.2 [PDF 1.2], HEVEIN-LIKE/PATHOGENESIS-RELATED 4 [HEL $P R 4])$. Cycle threshold values of defense genes were normalized to the geometric mean of two housekeeping genes, namely ACT8 and $S A N D$, and the transcription of both genes is shown in Supplementary Figure S1. A list of analyzed genes and primers is available in Supplementary Table S1. Furthermore, to visualize the activation of marker genes on a cellular level in different leaf tissues surrounding the stylet insertions, we used a set of promoter-reporter transgenic lines of A. thaliana (Marhavý et al. 2019), expressing fusions of defense gene promoters with mVenus reporter and a nuclear localization signal (NLS). The lines were originally designed to study gene activation in roots during infestation by the nematode Heterodera schachtii. Hence, the authors monitored nematode performance from the first moment of root invasion until the feeding establishment and analyzed SA, JA, and ET-associated responses. During this cellular damage interval, the induction of SA and JA-associated markers was not detected, while the expression of ET marker was significantly induced. We found this method applicable for experiments with aphids, as with this approach the single aphid "effect zone" can be studied. We tested several lines to dissect activation of separate hormone-associated signaling pathways: ET (ACS6), JA (AOS and JAZ10), ET/JA (PR4/HEL), and SA $(P R l)$. Unfortunately, high background noise was observed in noninfested leaves with reporter constructs for ACS6, AOS, and $P R 4 / H E L$, which brought difficulties for data interpretation (Supplementary Fig. S2). Reporter lines for SA (PR1) and JA (JAZ10) were found suitable for our system.

Aphid feeding caused upregulation of several genes involved in defense signaling and biosynthesis of defense-related hormones, as found by our qRT-PCR analysis of leaf discs at feeding sites (Fig. 1). Changes in the expression of one gene involved in SA synthesis (ICS1) and stress-responsive SA-related genes (PRI, $P R 2, P R 5)$ were detected at $24 \mathrm{~h}$ after $B$. brassicae infestation compared with noninfested control. ICSI showed fivefold induction at $24 \mathrm{hpi}$, while ICS 2 was significantly induced only from 48 hpi, suggesting this gene is involved in maintaining rather than in the establishment of the response. Transcription of PRI was over 100-fold higher in aphid-infested leaf discs at 24 hpi compared with noninfested control and about 1,000-fold higher at 48 and 72 hpi (Fig. 1). As for PR2 and PR5, the pattern of induction was similar to that of $P R 1$ (gradual increase in transcription starting from $24 \mathrm{hpi}$ ). Previous studies also reported strong induction of $P R I$ gene 72 hpi by a specialist aphid (Kuśnierczyk et al. 2007, 2008; Moran et al. 2002). However, PRl fold change was considerably higher in our study, likely because we analyzed leaf discs closely surrounding the feeding sites while other researchers analyzed whole leaves or even rosettes. Kuśnierczyk et al. (2007) infested three different ecotypes of $A$. thaliana (Ws, Cvi, and Ler) with $B$. brassicae and, after harvesting the whole leaves, the induction of PRI as a consequence of aphid infestation was five-, two-, and 21-fold in Ws, Cvi, and Ler ecotype, respectively. Moreover, Moran et al. (2002) reported 23-fold induction of $P R 1$ in infested leaves of $A$. thaliana ecotype Col-0 at 72 hpi. Surprisingly, Onkokesung et al. (2019) observed the induction of PRI while sampling whole rosettes of $A$. thaliana ecotype Col-0 (up to 50-fold) already at $6 \mathrm{~h}$ after infestation with $B$. brassicae; however, no induction was observed at 24, 48, and $72 \mathrm{hpi}$. The significant and strong induction at later timepoints found in our study could be caused by our spatial sampling technique, as, for the whole leaf and rosette sampling, the signal may be masked by the background level of many noninduced cells.

In addition to the SA-related marker genes, our qRT-PCR analysis found an early induction of several ET and JA marker genes in leaf tissues surrounding feeding sites. Infestation triggered the expression of the gene coding for the ZIM-domain protein, $J A Z 8$, already at $6 \mathrm{hpi}$ and strongly increased the expression of JAZ8 and JAZ10 genes at 24 and 72 hpi (Fig. 1). On the contrary, genes involved in the octadecanoid signaling pathway or wound-inducible JA pathway ( $L O X 2, A O S)$ were significantly upregulated in aphid-infested samples at 24 and 48 hpi. On the other hand, Kuśnierczyk et al. (2007) observed rather low induction of JA biosynthetic genes in the whole leaves of different ecotypes of Arabidopsis only at $72 \mathrm{hpi}$; the change in $L O X 2$ expression ranged from three- to sixfold and, in AOS expression, from two- to threefold. Later on, Kuśnierczyk et al. (2008) also reported lower but still significant induction of JAZ10 gene in the whole rosettes of A. thaliana ecotype Ler at 6,24, and 48 hpi (up to 1.4-fold).

As for the genes important for ET synthesis, ACS2 and ACS6, our qRT-PCR analysis showed the induction already at $6 \mathrm{hpi}$, indicating an early response to $B$. brassicae attack; then, low but significant induction was observed at $24 \mathrm{hpi}$, followed by the strong upregulation at 48 and 72 hpi (Fig. 1). Our results are in line with other studies reporting the involvement of ET responses during aphid infestations in whole leaves and rosettes (Kuśnierczyk et al. 2007, 2008; Smith and Boyko 2007). Kuśnierczyk et al. (2007) also observed an increase in ACS6 transcription in B. brassicae-infested leaves of $A$. thaliana ecotype Ws (up to twofold), Cvi (up to 13-fold), and Ler (up to twofold) at $72 \mathrm{hpi}$. In our setup, genes involved in both JA and ET signaling pathways, $P R 4 / H E L$ and PDF1.2, were also found to be induced in infested leaf discs at 24, 48, and 72 hpi (Fig. 1). Interestingly, Moran et al. (2002) reported higher induction of PDF1.2 in B. brassicaeinfested leaves of $A$. thaliana ecotype Col-0 (up to 48-fold) after 72 h of aphid feeding, while Kuśnierczyk et al. (2008) 
PR1

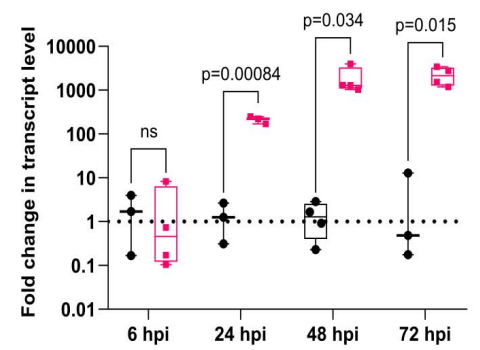

ICS1

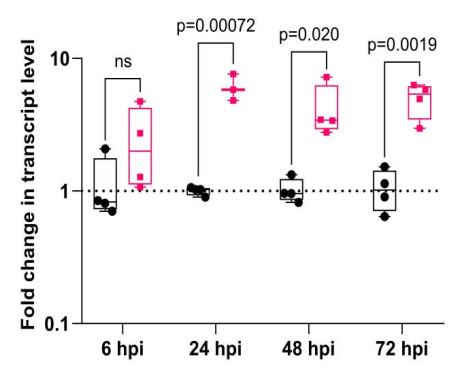

LOX2

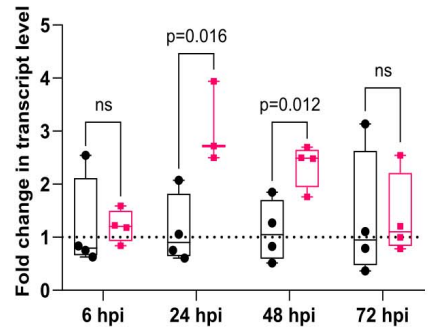

ACS2

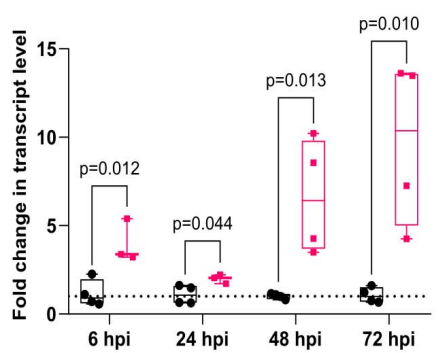

HEL/PR4

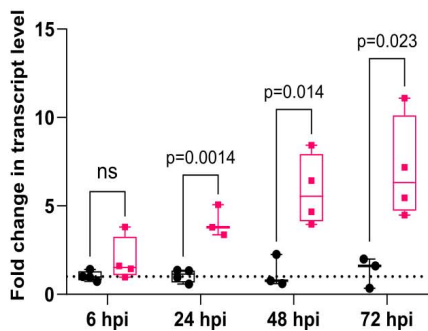

PR2

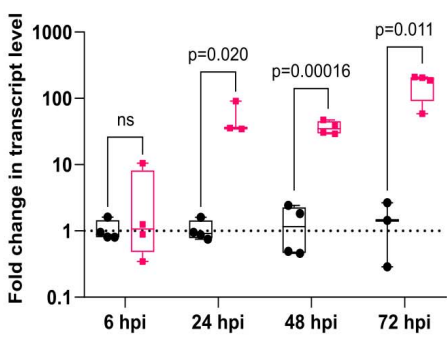

ICS2

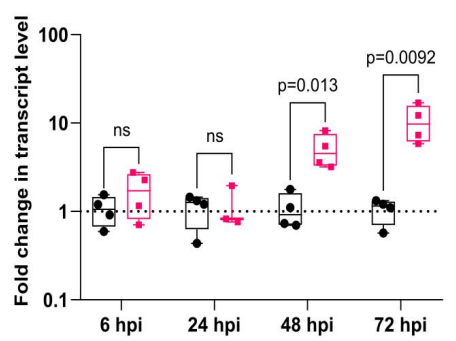

JAZ8

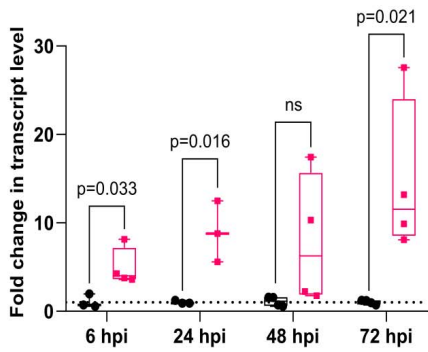

ACS6

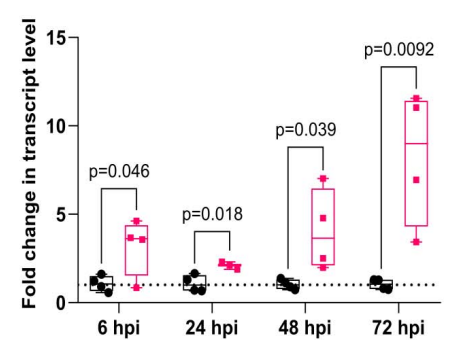

PR5

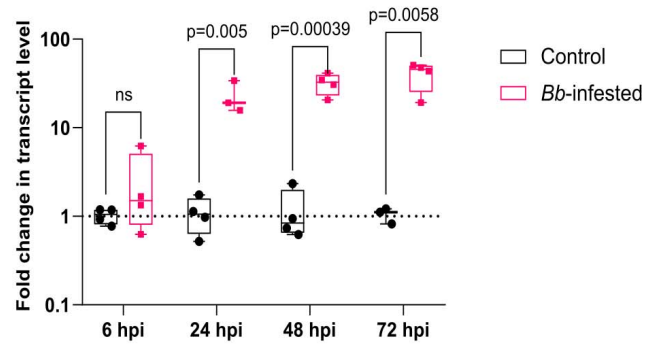

AOS

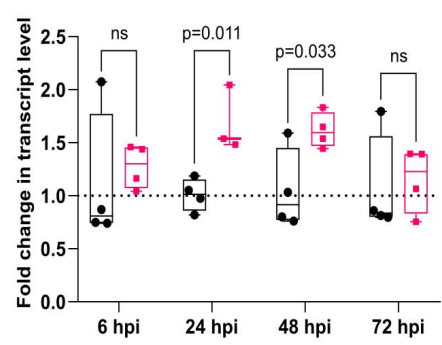

JAZ10

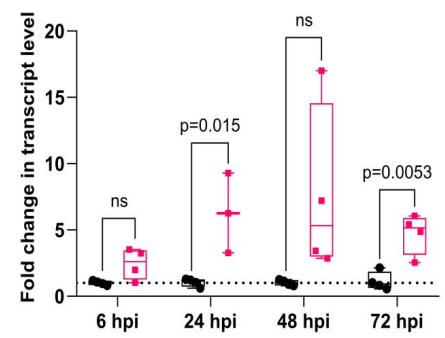

PDF1.2

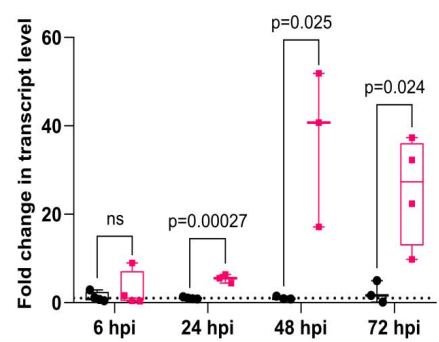

Fig. 1. Gene expression changes in leaf discs around feeding sites of the specialist aphid Brevicoryne brassicae in wild-type plants of Arabidopsis thaliana (Col-0) after 6, 24, 48, and $72 \mathrm{~h}$ of infestation. Graphs represent genes involved in the salicylic acid signaling pathway (PR1, PR2, PR5, ICS1, ICS2), jasmonic acid (JA) signaling pathway ( $L O X 2, A O S, J A Z 8, J A Z 10)$, ethylene (ET) biosynthetic pathway (ACS2, ACS6), and ET/JA signaling pathway $(P D F 1.2, H E L / P R 4)$. Cycle threshold values of target genes were normalized to the geometric mean of two housekeeping genes, namely $A C T 8$ and $S A N D$. Control and infested samples were compared within each timepoint with the Student's $t$ test; $P$ value is indicated for comparisons that passed the significance threshold; normality of data distribution was checked using the Shapiro-Wilk test; $n=4$. Error bars represent standard error of the mean. Dashed line on $y=1$ is set to a normalized mean of noninfested control for each timepoint. 
reported lower induction of $P D F 1.2$ in infested rosettes of Ler ecotype 48 hpi (up to twofold). Similarly to our results, Kuśnierczyk et al. (2007, 2008) reported gradual upregulation of the HEL gene in the leaves and whole rosettes of $A$. thaliana ecotype Ler at 6, 24, 48, and $72 \mathrm{hpi}$; however, the induction was relatively low (up to 2.7 -fold).

Using fluorescent reporter lines, we were also able to detect an induced transcription of $P R I$ and JAZ1O in the cells surrounding the stylet puncture at $24 \mathrm{hpi}$ in comparison with noninfested plants (Fig. 2A and B). Notably, the induction started from the sites of stylet punctures (visible as an autofluorescent line [Fig. 2B]) at $24 \mathrm{hpi}$, and, until $48 \mathrm{hpi}$, was spreading along the veins, while, at $72 \mathrm{hpi}$, the reporter fluorescence was detected also in the leaf epidermis pavement cells outside the vein (Fig. 2B). The induction of PRI and JAZ1O was evaluated by manual counting of the number of cells with nuclear fluorescence per leaf disc. Statistically significant induction of $P R 1$ and JAZ1O was detected in the cells of aphid-infested plants at each timepoint (Fig. 2C).
A

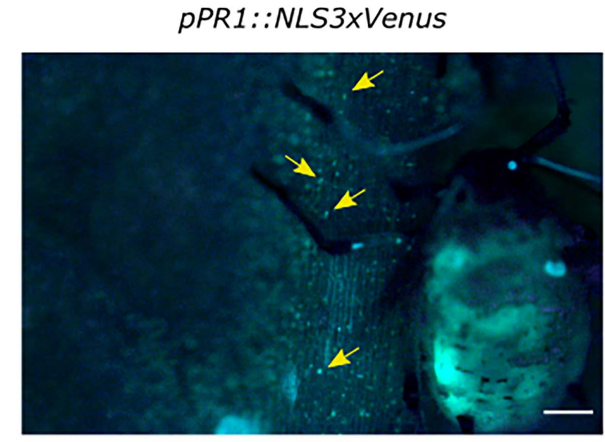

pJAZ10::NLS3XVenuS

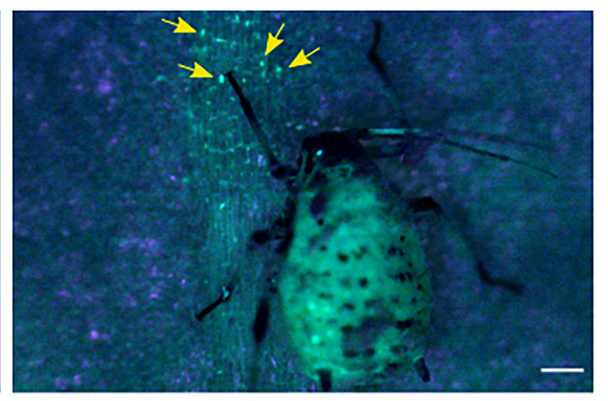

B control $24 \mathrm{hpi}$ 48 hpi 72 hpi

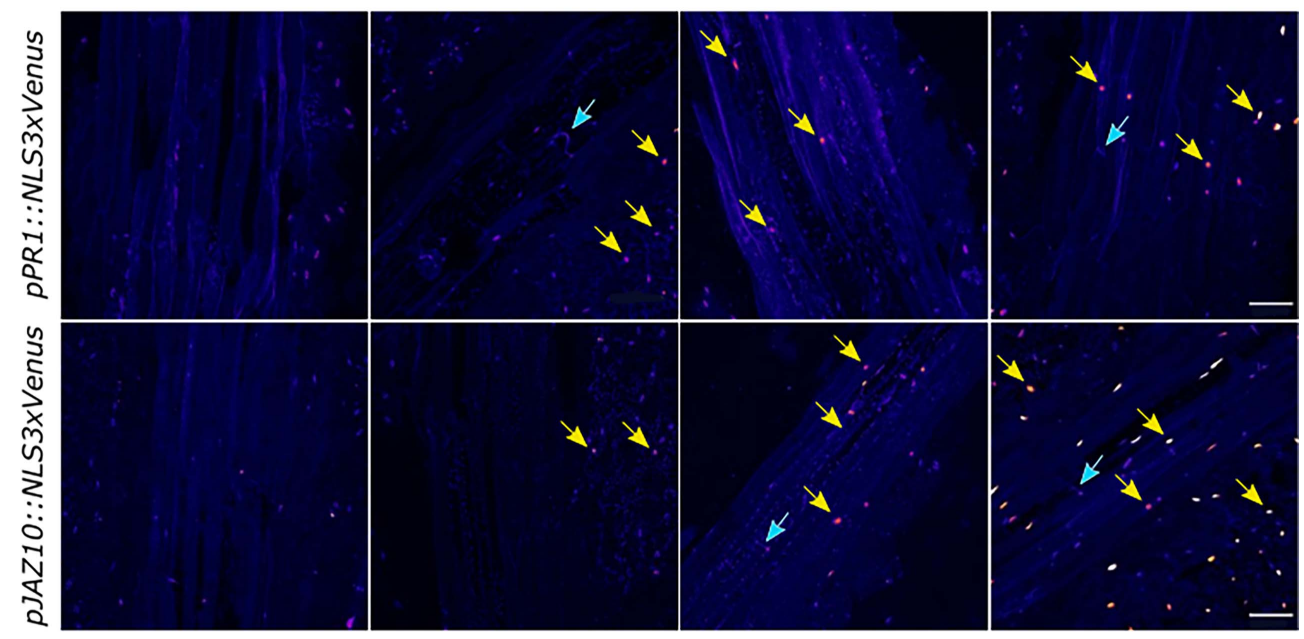

C
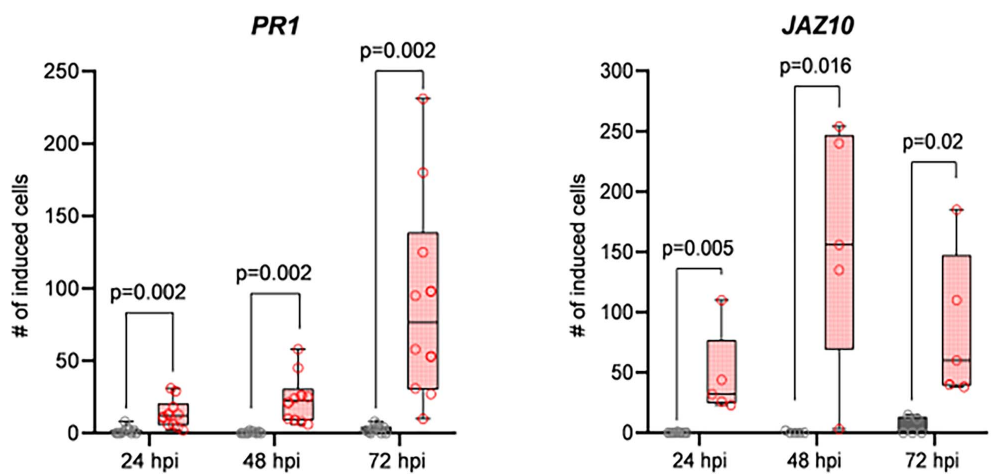

Fig. 2. Local activation of $P R 1$ and JAZ10 transcription in Arabidopsis thaliana veins upon infestation around the feeding sites of Brevicoryne brassicae. A, B. brassicae feeding on the leaves of $p P R 1:: N L S 3 x$ Venus and $p J A Z 10:: N L S 3 x$ Venus $48 \mathrm{~h}$ postinfestation (hpi). Scale bar $=500 \mu \mathrm{m} . \mathbf{B}$, Representative images of $P R 1$ and JAZ10 induction. Yellow arrows point to the nuclei with induced reporter construct expression, blue arrows point to the stylet puncture autofluorescence. Scale bar $=10 \mu \mathrm{m}$. C, $P R 1$ and JAZ10 quantification of induced cells per 6-mm leaf disc. Control and infested samples were compared within each timepoint with the Student's $t$ test; normality of data distribution was checked using the Shapiro-Wilk test; $P$ value is indicated for comparisons that passed the significance threshold; $n=10$. Error bars represent standard error of the mean. 
To complement previous results regarding visualization of spatio-temporal dispersion of $P R I$ induction from the sites of aphid feeding, Arabidopsis plants expressing PR1::GUS were infested and analyzed by X-GLUC assay sampling whole infested leaves (Supplementary Methods S1). As expected, $\beta$-glucuronidase (GUS) activity was detected, as the PR1 gene was slightly induced in infested plants at $24 \mathrm{hpi}$ and more strongly in later timepoints (Fig. 3A and B) and was detectable around the
A control
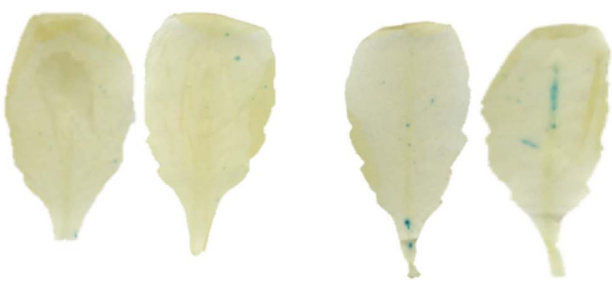

B
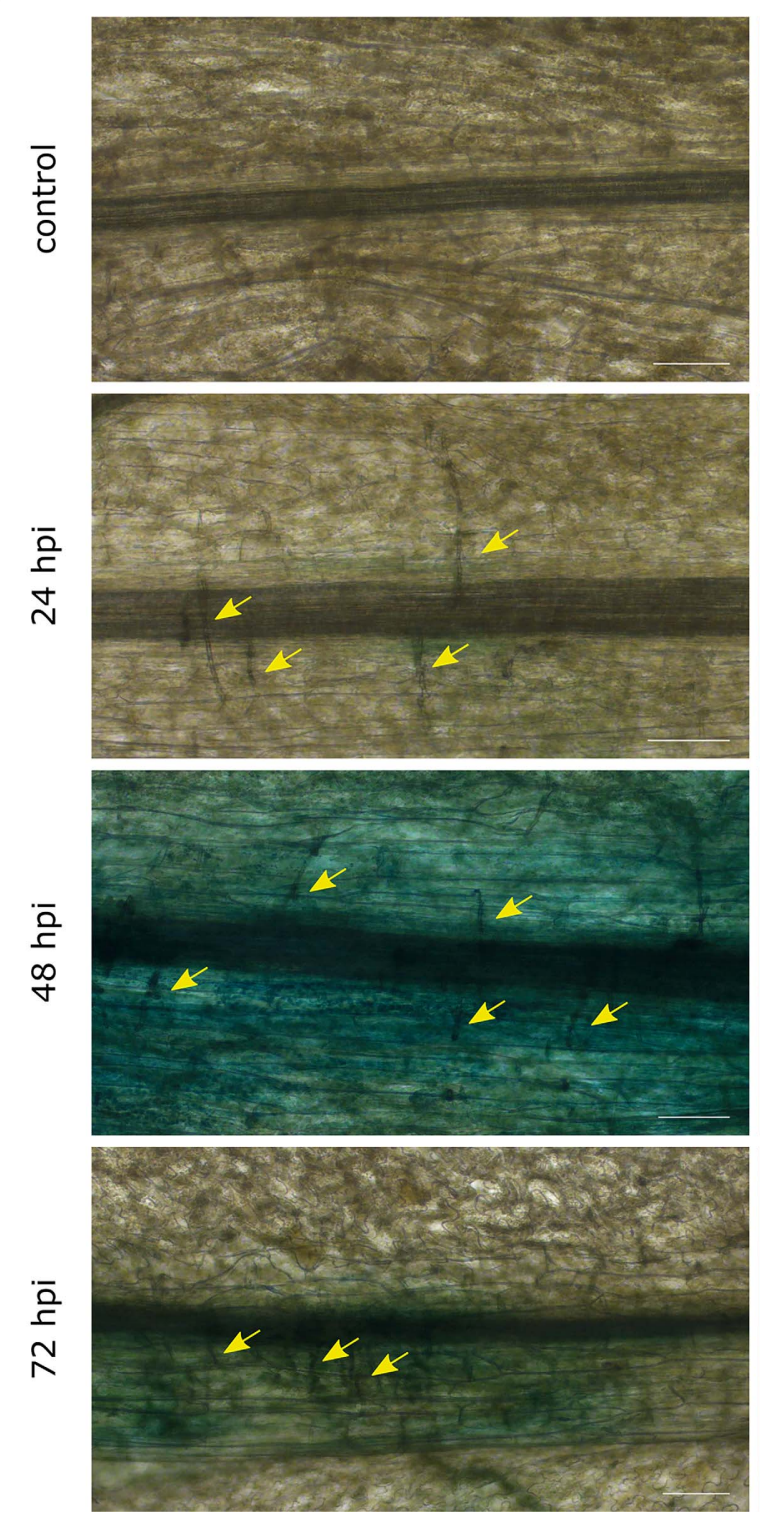

$48 \mathrm{hpi}$
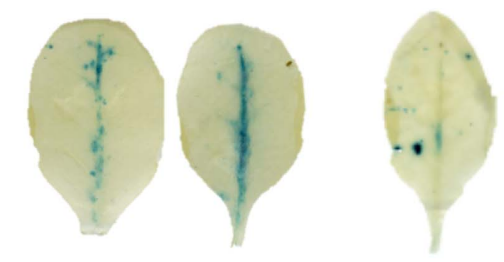

72 hpi

\section{Callose deposition}
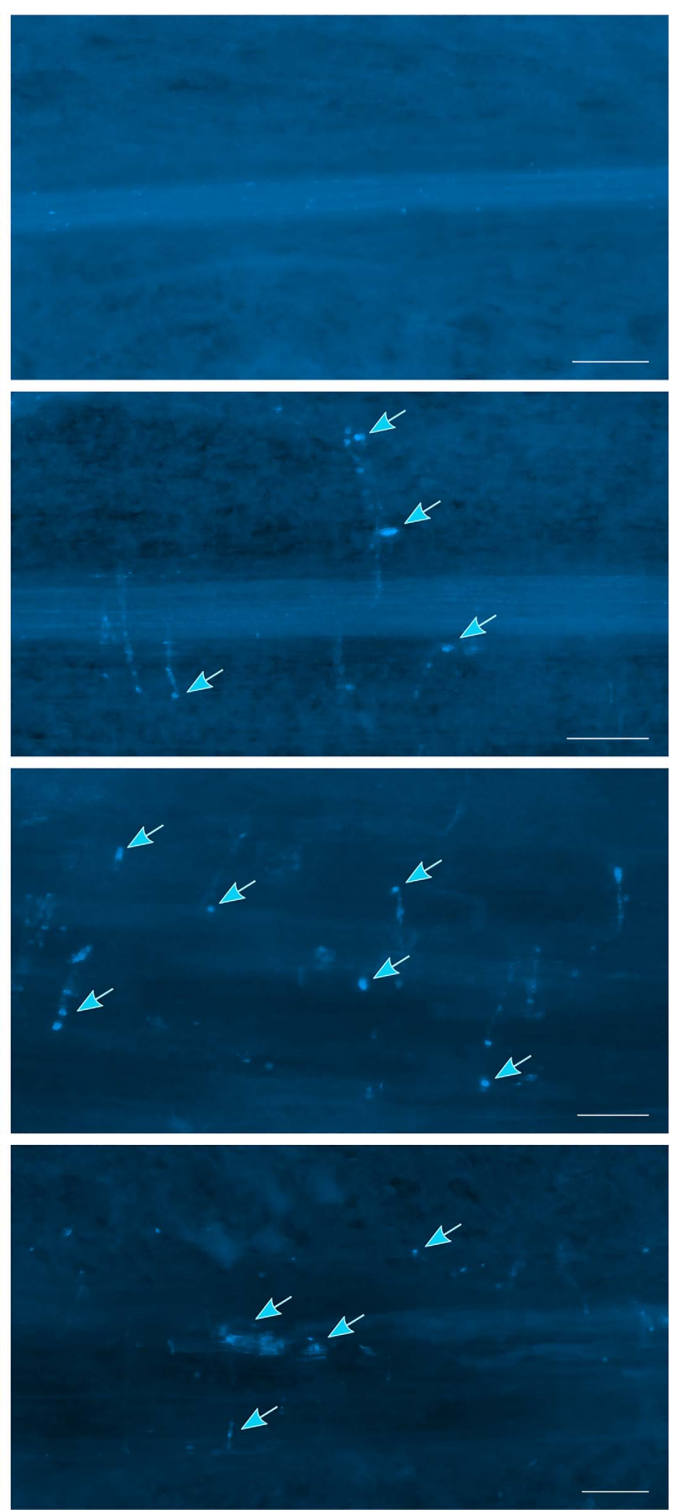

Fig. 3. Brevicoryne brassicae triggers $P R 1$ expression and callose deposition. A, Histochemical staining of $\beta$-glucuronidase (GUS) activity in leaves of PR1::GUS at 24, 48, and $72 \mathrm{~h}$ postinfestation (hpi) by B. brassicae. B, Dynamics of PR1::GUS expression and callose deposition in leaves at 24, 48, and 72 hpi by B. brassicae, Yellow arrows indicate the sites of stylet insertion, blue arrows point to deposited callose. Scale bar $=100 \mu \mathrm{m}$. 
sites of stylet puncturing (Fig. 3B, yellow arrows). Indeed, the strong induction of $P R 1$ seems to occur locally around the aphid feeding zones), as we found both while sampling leaf discs for qRT-PCR analysis (Fig. 1) and when using promoter-reporter line pPR1::NLS3xVenus (Fig. 2A and B).

Callose, a $\beta$-glucan polymer synthesized at the plasma membrane of plant cells, plays an important role in plant defense responses to aphid infestation (Silva-Sanzana et al. 2020). To study whether callose deposition is spatially connected with defense gene activation, we subsequently stained $P R 1:: G U S$ leaves with aniline blue for callose deposition analysis after X-GLUC assay (Supplementary Methods S1). Callose deposition was detected at all studied timepoints at the sites of $B$. brassicae stylet insertions; hence, it colocalized with $P R I$ gene activation (Fig. 3B). Our findings confirmed previously observed callose formations (Kuśnierczyk et al. 2008) around the stylet insertion sites during B. brassicae wounding of Arabidopsis cells. Interestingly, generalist aphids seem to trigger similar responses in Arabidopsis. De Vos et al. (2005) observed PRl gene induction around aphid feeding sites in PR1::GUS-expressing leaves but only after $72 \mathrm{~h}$ of Myzus persicae feeding. This could be explained by variation in protein concentration and composition of saliva between specialist and generalist aphid species that causes different response intensity from plant defense systems (Will et al. 2009).

With the development of novel visualization tools, the research on plant-pest interactions is getting more precise and detailed. We show that the stylet penetration during attack by the $B$. brassicae aphid causes a rapid activation of plant defense signals in the cells surrounding the stylet puncture in A. thaliana. In time, the induction spreads along the veins and, to a lesser extent, radially to the leaf tissues. In this work, we particularly show that subtle, though biologically important, changes in transcriptomic response can be detected by performing precise sampling procedures (i.e., collecting leaf discs around feeding sites instead of pooling material from the whole infested rosettes or leaves). Local transcriptomic remodeling seems to occur faster in tissues close to feeding sites than in the rest of the leaf when compared with previously published studies. Our study therefore also suggests that important dynamics in space and time soon after herbivore attack may be missed if not using spatially explicit sampling, as also found for the reaction of leaf photosynthesis to herbivory (Moustaka et al. 2021). We wish to draw attention to the need for a detailed description of sampling protocols that might influence data output and drive conclusions, especially in high-throughput methods like transcriptomics or metabolomics.

\section{ACKNOWLEDGMENTS}

We thank N. Geldner for kindly providing us with reporter lines of Arabidopsis thaliana and K. Malínská for helping with microscopic observations and imaging.

\section{LITERATURE CITED}

De Vos, M., Van Oosten, V. R., Van Poecke, R. M. P., Van Pelt, J. A., Pozo, M. J., Mueller, M. J., Buchala, A. J., Métraux, J.-P., Van Loon, L. C., Dicke, M., and Pieterse, C. M. J. 2005. Signal signature and transcriptome changes of Arabidopsis during pathogen and insect attack. Mol. Plant-Microbe Interact 18:923-937.

Jaouannet, M., Rodriguez, P. A., Thorpe, P., Lenoir, C. J. G., MacLeod, R., Escudero-Martinez, C., and Bos, J. I. B. 2014. Plant immunity in plant-aphid interactions. Front. Plant Sci. 5:663.

Knoblauch, M., and van Bel, A. J. E. 1998. Sieve tubes in action. Plant Cell 10:35-50.

Kuśnierczyk, A., Winge, P., Jørstad, T. S., Troczyńska, J., Rossiter, J. T. and Bones, A. M. 2008. Towards global understanding of plant defence against aphids-Timing and dynamics of early Arabidopsis defence responses to cabbage aphid (Brevicoryne brassicae) attack. Plant Cell Environ. 31:1097-1115.

Kuśnierczyk, A., Winge, P., Midelfart, H., Armbruster, W. S., Rossiter, J. T., and Bones, A. M. 2007. Transcriptional responses of Arabidopsis thaliana ecotypes with different glucosinolate profiles after attack by polyphagous Myzus persicae and oligophagous Brevicoryne brassicae. J. Exp. Bot. 58:2537-2552.

Marhavý, P., Kurenda, A., Siddique, S., Dénervaud Tendon, V., Zhou, F., Holbein, J., Hasan, M. S., Grundler, F. M., Farmer, E. E., and Geldner, N. 2019. Single-cell damage elicits regional, nematoderestricting ethylene responses in roots. EMBO J. 38:e100972.

Mewis, I., Appel, H. M., Hom, A., Raina, R., and Schultz, J. C. 2005. Major signaling pathways modulate Arabidopsis glucosinolate accumulation and response to both phloem-feeding and chewing insects. Plant Physiol. 138:1149-1162.

Miles, P. W. 1959. Secretion of two types of saliva by an aphid. Nature 183:756.

Miles, P. W. 2007. Aphid saliva. Biol. Rev. 74:41-85.

Moran, P. J., Cheng, Y., Cassell, J. L., and Thompson, G. A. 2002. Gene expression profiling of Arabidopsis thaliana in compatible plant-aphid interactions. Arch. Insect Biochem. Physiol. 51:182-203.

Moran, P. J., and Thompson, G. A. 2001. Molecular responses to aphid feeding in Arabidopsis in relation to plant defense pathways. Plant Physiol. 125:1074-1085

Moustaka, J., Meyling, N. V., and Hauser, T. P. 2021. Induction of a compensatory photosynthetic response mechanism in tomato leaves upon short time feeding by the chewing insect Spodoptera exigua. Insects 12:562.

Onkokesung, N., Reichelt, M., Wright, L. P., Phillips, M. A., Gershenzon, J., and Dicke, M. 2019. The plastidial metabolite 2- $C$-methyl- $D$-erythritol2,4-cyclodiphosphate modulates defence responses against aphids. Plant Cell Environ. 42:2309-2323.

Silva-Sanzana, C., Estevez, J. M., and Blanco-Herrera, F. 2020. Influence of cell wall polymers and their modifying enzymes during plant-aphid interactions. J. Exp. Bot. 71:3854-3864.

Smith, C. M., and Boyko, E. V. 2007. The molecular bases of plant resistance and defense responses to aphid feeding: Current status. Entomol. Exp. Appl. 122:1-16.

Tjallingii, W. F. 2006. Salivary secretions by aphids interacting with proteins of phloem wound responses. J. Exp. Bot. 57:739-745.

Will, T., Kornemann, S. R., Furch, A. C. U., Tjallingii, W. F., and van Bel, A. J. E. 2009. Aphid watery saliva counteracts sieve-tube occlusion: A universal phenomenon? J. Exp. Biol. 212:3305-3312.

Will, T., Tjallingii, W. F., Thönnessen, A., and van Bel, A. J. E. 2007. Molecular sabotage of plant defense by aphid saliva. Proc. Natl. Acad. Sci. U.S.A. 104:10536-10541. 